\title{
LETTERS
}

\section{Small airways in asthma: their independent contribution to the severity of hyperresponsiveness}

\section{To the Editor:}

Bronchial hyperresponsiveness (BHR), i.e. increased narrowing of the airways after exposure to non-allergic stimuli, is a hallmark of asthma. BHR is a risk factor for asthma development and, additionally, a marker of worse disease outcome in asthma [1, 2]. It is generally acknowledged that obstruction of the large airways due to inflammation and remodelling contributes to more severe BHR. This is plausible, given that $\mathrm{BHR}$ is expressed as the concentration or dose of a stimulus that induces a $20 \%$ fall in forced expiratory volume in $1 \mathrm{~s}$ (FEV1). Recent studies suggest that asthmatics with BHR have more severe small airways obstruction [3,4]. However, little is known about the converse, i.e. the association between small airways obstruction and the severity of BHR. Our aim was to assess: 1) whether asthma patients with small airways obstruction express more severe BHR than those without small airways obstruction; and 2) whether small airways obstruction is associated with more severe BHR independently of FEV1.

We analysed data from patients with mild-to-moderate asthma who were included in a previously published study on inhaled corticosteroids (ICS) in primary care [5]. All subjects underwent spirometry before and after $1 \mathrm{mg}$ terbutaline, measuring FEV1, forced vital capacity (FVC) and mean expiratory flow at $50 \%$ of FVC (MEF50). BHR was assessed using a histamine challenge test, measuring the provocative dose causing a 20\% fall in FEV1 (PD20) (histamine). All patients were hyperresponsive to histamine (PD20 $<9 \mathrm{mg}$ ). Small airways obstruction was defined as a MEF50 of less than or the same as the lower limit of normal (LLN); other small airways parameters, such as the mean expiratory flow between 25-75\% of FVC (MEF25-75), residual volume/total lung capacity and FVC/slow vital capacity, were not available in our database. We compared asthma patients with and without small airways obstruction using unpaired ttests, Mann-Whitney U-tests or Chi-squared tests as appropriate. In addition, a multivariate linear regression analysis was performed to assess whether MEF50 is independently associated with the PD20 histamine ( $\log _{2}$ transformed). We added FEV1, age, sex, height and use of ICS as covariates.

Of the 94 patients, 34 had small airways obstruction (MEF50 $\leqslant$ LLN). Table 1 shows that patients with small airways obstruction had more severe BHR (geometric mean PD20 histamine 0.2 versus $0.6 \mathrm{mg}$ respectively, $\mathrm{p}<0.01$ ). In addition, FEV1, FVC and FEV1/FVC values were significantly lower and reversibility values were significantly higher in patients with small airways obstruction. Patients with small airways obstruction using ICS had a significantly higher daily dose of ICS than patients without small airways obstruction (800 versus $500 \mu \mathrm{g}$ per day beclomethasone dipropionate equivalent). A lower MEF50 value was significantly associated with more severe
BHR in the multivariate linear regression analysis, independently from FEV1, age, sex, height and ICS use $(b=0.813$, $\mathrm{p}=0.01$ ). We repeated this analysis with MEF50/FVC instead of MEF50, and with MEF50 \% predicted and FEV1 \% pred instead of the absolute values. These analyses showed similar results.

This study shows that asthma patients with small airways obstruction have more severe BHR independently of the level of FEV1. Despite intensive research over the past 40 years, the mechanisms underlying BHR have still not been clarified. It is known that the severity of BHR is related to airway wall inflammation in asthma. Other factors that influence BHR are the structural changes of the airways such as deposition of connective tissue components, increased vessel formation and smooth muscle mass and oedema, contributing to thickening and increased stiffness of the airways. These processes are known to occur in the large airways and there is now increasing evidence to suggest that they also occur in the small airways. Small airways obstruction, i.e. lower MEF25-75 values, has previously been reported to be present in children with BHR and in adults with mild asthma and BHR [3, 4]. Another study showed that patients with hyperresponsiveness to mannitol have more severe small airways obstruction [6]. Together, these and our results suggest that an unexplained part of BHR originates from the small airways.

A limitation of our study may be the use of MEF50 as a marker of small airways obstruction. Obstruction in the proximal airways may also lead to airflow limitation with reduced MEF50. As the patients with a MEF50 $\leqslant$ LLN had a reduced FEV1, we performed a multiple linear regression analysis in which we corrected for FEV1. In this analysis, MEF50 was associated with the severity of BHR independently of FEV1. In addition, we performed our analysis with MEF50/FVC, since FVC is known to influence MEF50 levels, and with MEF50 \% pred and FEV1 \% pred. The results remained similar, demonstrating the robustness of our findings.

We found that small airways obstruction is associated with the severity of BHR in asthma, independently of FEV1, and this may have important implications for asthma management. Treatment with large-particle ICS rapidly improves FEV1 in asthma, an effect that remains stable thereafter. In contrast, improvement in BHR occurs gradually and ICS do not completely abolish the presence of BHR in most asthmatics, even after longstanding treatment [7]. This may have implications for asthma management; SONT et al. [8] showed that ICS treatment tailored according to the severity of BHR improves the clinical outcome and airway remodelling of asthma. In addition, targeting the small airways with currently available small-particle ICS may be more effective in reducing BHR originating in the small airways. A recent study demonstrated 
TABLE 1 Differences between asthma patients with and without small airways obstructions

\begin{tabular}{|c|c|c|c|}
\hline & MEF50 > LLN & MEF50 $\leqslant$ LLN & p-value \\
\hline Subjects $n$ & 60 & 34 & \\
\hline Males & $22(37)$ & $13(38)$ & 0.88 \\
\hline Smoking & & & 0.39 \\
\hline Non-smoker & $30(51)$ & $21(62)$ & \\
\hline Habitual smoker & $7(12)$ & $2(6)$ & \\
\hline Pack-yrs ${ }^{\#}$ & $3.0(1.0-6.0)$ & $4.0(1.0-6.0)$ & 0.96 \\
\hline ICS use & $54(90)$ & $31(91)$ & 0.85 \\
\hline ICS dose BDP equivalent ${ }^{*} \mu \mathrm{g} \cdot$ day $^{-1}$ & $500(400-800)$ & $800(400-800)$ & $<0.01$ \\
\hline $\mathrm{BMI} \mathbf{k g} \cdot \mathrm{m}^{-2}$ & $26(24-30)$ & $26(24-30)$ & 0.69 \\
\hline PD20 histamine $\mathbf{m g}^{+}$ & $0.6(0.1-4.2)$ & $0.2(0.1-2.7)$ & $<0.01$ \\
\hline Reversibility \% pred & $4.9(3.0-7.5)$ & $8.4(5.6-11.1)$ & $<0.01$ \\
\hline MEF50 \% pred & $85.8(66.9-100.6)$ & $45.3(38.9-51.1)$ & $<0.01$ \\
\hline
\end{tabular}

Data are presented as median (interquartile range) or $\mathrm{n}(\%)$, unless stated otherwise. Patients with small airways obstruction were defined as having a mean expiratory flow at $50 \%$ of forced vital capacity (MEF50) that was less than or the same as the lower limit of normal (LLN). Patients without small airways obstruction were defined as having a MEF50 greater than the LLN. ICS: inhaled corticosteroids; BDP: beclomethasone dipropionate; BMl: body mass index; PD20: provocative dose causing a 20\% fall in forced expiratory volume in $1 \mathrm{~s}$ (FEV1); \% pred: \% predicted; FVC: forced vital capacity. ${ }^{*}$ : does not include non-smokers, $\because$ : only in patients using ICS, ${ }^{+}$: data are presented as geometric mean (range).

that treatment with small-particle ICS is at least equally effective in achieving asthma control as large-particle ICS treatment at half the dose [9]. Moreover, we have recently shown that smallparticle ICS induces a greater improvement of BHR to AMP than large-particle ICS, but only when the provocation was performed using small-particle AMP (1.74 versus 0.8 doubling doses, respectively) [10]. Taken together, our findings suggest that the currently applied provocation tests have important shortcomings for investigating BHR originating from the small airways. First, the read-out for airway obstruction during provocation is $\mathrm{FEV} 1$, which mainly a large airways parameter. Secondly, the DeVilbiss 646 (DeVilbiss Healthcare, Somerset, PA, USA) is the recommended nebuliser for provocation tests; this nebuliser produces relatively large particles of $3.7 \mu \mathrm{m}[10]$. Therefore, most particles will deposit in the large airways and few particles will actually reach the small airways. Thus, current provocation tests will underestimate the BHR of small airways. This is important, because BHR of the small airways may contribute to asthma symptoms and control in real life.

We conclude that the small airways contribute significantly to the severity of BHR, a contribution that is independent of the level of FEV1. We hypothesise that the contribution of the small airways to the severity of BHR in asthma is much larger than has been considered to date. Performing studies with provocation tests with small airways measurements, e.g. impulse oscillometry, and small-particle stimuli will give better insights into BHR of the small airways. Further studies should show whether small-particle ICS improves BHR more rapidly and/or to a larger extent than large-particle ICS, with the ultimate benefit being to asthma patients in clinical practice.
Eef D. Telenga*,\#, Maarten van den Berge ${ }^{*, \#}$, Nick H.T. ten Hacken $^{*, \#, \text { Roland A. Riemersma }}{ }^{\#, \text { I }}$, Thys van der Molen ${ }^{\#, \text {, }}$ Dirkje S. Postma ${ }^{*, \#,}$

*Dept of Pulmonary Diseases, University of Groningen, University Medical Center Groningen, 'Dept of General Practice, University of Groningen, University Medical Center Groningen, and \#GRIAC Research Institute, University of Groningen, University Medical Center Groningen, Groningen, The Netherlands.

Correspondence: D.S. Postma, University Medical Center Groningen (AA11), P.O. Box 30.001, 9700 RB Groningen, The Netherlands. E-mail: d.s.postma@umcg.nl

Statement of Interest: Statements of interest for M. van den Berge and D.S. Postma can be found at www. erj.ersjournals.com/site/ misc/statements.xhtml

\section{REFERENCES}

1 Jansen DF, Schouten JP, Vonk JM, et al. Smoking and airway hyperresponsiveness especially in the presence of blood eosinophilia increase the risk to develop respiratory symptoms: a 25-year follow-up study in the general adult population. Am J Respir Crit Care Med 1999; 160: 259-264.

2 Peat JK, Woolcock AJ, Cullen K. Rate of decline of lung function in subjects with asthma. Eur J Respir Dis 1987; 70: 171-179.

3 Simon MR, Chinchilli VM, Phillips BR, et al. Forced expiratory flow between $25 \%$ and $75 \%$ of vital capacity and FEV1/forced vital capacity ratio in relation to clinical and physiological parameters 
in asthmatic children with normal FEV1 values. J Allergy Clin Immunol 2010; 126: 527-534.

4 Currie GP, Jackson CM, Lee DK, et al. Determinants of airway hyperresponsiveness in mild asthma. Ann Allergy Asthma Immunol 2003; 90: 560-563.

5 Riemersma RA, Postma DS, Van der Molen T. Budesonide/ formoterol maintenance and reliever therapy in primary care asthma management: effects on bronchial hyperresponsiveness and asthma control. Prim Care Respir J 2012; 21: 50-56.

6 Aronsson D, Tufvesson E, Bjermer L. Comparison of central and peripheral airway involvement before and during methacholine, mannitol and eucapnic hyperventilation challenges in mild asthmatics. Clin Respir J 2011; 5: 10-18.

7 Douma WR, Kerstjens HAM, de Gooijer A, et al. Initial improvements in lung function and bronchial hyperresponsiveness are maintained during 5 years of treatment with inhaled beclomethasone dipropionate and terbutaline. Chest 2002; 121: 151-157.

8 Sont JK, Willems LN, Bel EH, et al. Clinical control and histopathologic outcome of asthma when using airway hyperresponsiveness as an additional guide to long-term treatment. The AMPUL Study Group. Am J Respir Crit Care Med 1999; 159: 1043-1051.

9 Barnes N, Price D, Colice G, et al. Asthma control with extrafineparticle hydrofluoroalkane-beclometasone vs. large-particle chlorofluorocarbon-beclometasone: a real-world observational study. Clin Exp Allergy 2011; 41: 1521-1532.

10 Cohen J, Postma DS, Douma WR, et al. Particle size matters: diagnostics and treatment of small airways involvement in asthma. Eur Respir J 2011; 37: 532-540.

\section{Sarcoid-like lesion is a frequent benign cause of lymphadenopathy in neoplastic patients}

\section{To the Editor:}

Sarcoidosis is a systemic granulomatous disease of unknown origin, characterised by the widespread development of noncaseating epithelioid cell granulomas in more than one organ system. Clinical and radiographic aspects of sarcoidosis and malignancy might mimic one another, making the distinction between the two difficult in some cases. Cancer and sarcoidosis have been associated in some case series but this association remains controversial; several studies from the literature suggest that this association is not fortuitous [1, 2]. Sarcoidosis may precede, follow or occur concurrently with cancer, both haematological malignancies and solid tumours [2].

We wanted to evaluate the incidence of morphological features of sarcoidosis in patients followed up for previous cancer who develop hilar/mediastinal lymphadenopathies with no pulmonary lesions. We performed a retrospective chart review of all patients who were referred to our pulmonology department (Morgagni Hospital, Forlì, Italy) in the period between January 2007 and December 2011 with a new onset of hilar/mediastinal lymphadenopathies (with no pulmonary lesions) during follow-up for previous malignancies. Patients underwent endobronchial ultrasound (EBUS) or transoesophageal ultrasound (EUS) trans-bronchial needle aspiration (TBNA) under deep sedation (i.v. propofol). Lymphadenopathies were diagnosed $49 \pm 69$ months after the diagnosis of previous malignancy (minimal and maximal duration between cancer diagnosis and EBUS/EUS was 3 and 334 months, respectively). All pathology specimens were reviewed at our institute. Specimens were classified as inadequate (in the absence of lymphocytes) or diagnostic (in the presence of malignant cells, granulomas and mature lymphocytes). A granuloma was defined in this study as a discrete nodular aggregate of epithelioid histiocytes. In the case of inadequate samples, patients were addressed to standard cervical mediastinoscopy or video-assisted thoracoscopy for further investigations before any surgical treatment. In case of a positive cytological result of malignancy at EBUS-TBNA, patients were treated accordingly. The study was approved by the local institutional review board and all subjects gave written informed consent.

As shown in table 1, a total of 48 patients underwent EBUS/ EUS-TBNA; for all patients, the procedure was completed without problems and the feasibility of the procedure was $100 \%$. The mean patient age was 63 yrs (range $20-81 \mathrm{yrs}$ ); 31 patients were male and 17 were female. Patients had been followed-up for lung cancer $(n=10)$, lymphoma $(n=9)$, breast cancer $(n=7)$, urologic cancer $(n=6)$, gastrointestinal tumours $(n=6)$, larynx/pharynx tumours $(n=4)$, gynaecological tumours $(n=3)$ or other cancer $(n=8)$ (table 1$)$. Six patients had a history of two or more tumours in the past. None of the patients had suffered local recurrence or distant metastasis before the procedure was performed. Procedure was diagnostic in $45(94 \%)$ patients while inadequate specimens were obtained in three $(6 \%)$ patients. Among the 45 adequate samples, EBUS/EUS-TBNA showed non-necrotising granulomas with mature lymphocytes in a background suggestive of sarcoidosis in $12(26.7 \%)$ patients, lymph node metastases in 13 $(28.9 \%)$ patients and hyperplastic nonspecific lymphadenopathy in $20(44.4 \%)$ patients (table 1$)$. Negative samples were confirmed by surgical intervention in three cases while 17 patients were followed-up for 12 months; none of the patients suffered relapse or oncological events during the period of follow-up and all patients were alive and free of disease at the time of writing. Among the 12 patients with non-necrotising granulomas, 11 (92\%) patients were asymptomatic at the time of EBUS/EUS-TBNA and during the subsequent follow-up (average time 14 months); only one patient required steroid treatment for asthenia.

When the noncaseating epithelioid cell granuloma lesion of sarcoidosis is present but without accompanying systemic symptoms, this is referred to as a "sarcoid-like reaction" and is not distinguishable in terms of histopathological criteria [3]. 